\title{
Vertical Dynamic Response of Rock-Socketed Piles in a Layered Foundation
}

\author{
Chun Lin Liu ${ }^{1,2}$, Shuo Zhang ${ }^{1,2}$, Meng Xiong Tang ${ }^{1}$, He Song $\mathrm{Hu}^{1}$, Zhen Kun $\mathrm{Hou}^{1,2}$ and Hang Chen ${ }^{1}$ \\ ${ }^{1}$ Guangzhou Institute of Building Science Co., Ltd., 510440, Guangzhou, China \\ ${ }^{2}$ School of Civil Engineering and Transportation, South China University of Technology, 510641, Guangzhou, China
}

\begin{abstract}
A simplified method is presented to investigate the dynamic response of rock-socketed piles embedded in a layered foundation. The finite element method is utilized to derive the dynamic stiffness matrix equations of the pile modelled as a $1 \mathrm{D}$ bar, and the exact stiffness matrix method is employed to establish the flexibility matrix equations of the foundation modelled as a $3 \mathrm{D}$ body. According to the pilesoil interaction condition, these matrices are incorporated together to obtain the solution for the dynamic response of rock-socketed piles. Finally, some numerical results are given to illustrate the influence of rocksocketed depth on the pile vertical impedance.
\end{abstract}

\section{Introduction}

Pile foundations are widely used in the fields of building engineering, bridge engineering and offshore engineering, due to their higher bearing capacity and well stratigraphic adaptability. Many structures supported on the pile foundation are often subjected to dynamic loadings, such as the earthquake loading, traffic loading and machine loading. In some cases, these dynamic loadings would lead to the decrease in the pile bearing capacity (namely dynamic impedance) and would further result in the failure of the superstructures supported on pile foundations. Therefore, various methods have been proposed in the past decades to analyze the dynamic response of single piles or pile groups under dynamic conditions.

Based on the Lagrange's equation and discretization method, Rajapakse and Shah [1] studied the vertical vibration of a pile in an elastic half-space. A combination of Green's functions and fictitious bar method, Rajapakse and Shah [2] further carried out a parametric study on the dynamic characteristics of single piles embedded in an elastic medium. Using the same method, Zeng and Rajapakse [3] discussed the effect of length/radius ration and excitation frequency on the dynamic response of the pile embedded in a poroelastic soil layer, and $\mathrm{Lu}$ et al. [4] analyzed the vertical impedance of single piles in layered saturated media and concluded that the softer soil layer near the pile shaft has significant effect on the pile impedance. FEM is commonly considered to be an effective tool for establishing the complex dynamic interaction equations of the pile and the soil, Liu and Novak [5] and Zheng [6] utilized the FEM to investigate the dynamic behaviour of the pile in layered soils with Green' $\mathrm{s}$ functions for the soil flexibility matrices. In addition, a coupling method of BEM and FEM was employed by some researchers $[7,8]$ to analyze the dynamic characteristics of pile-soil interaction system in order to estimate the dynamic stiffness and damping of the pile foundation. Recently, a inhomogeneous pile subjected to a dynamic load acting on the pile head was analyzed by Wang et al. [9]; the harmonic response of an embedded single pile under dynamic conditions was carried out by Ai and Liu [10] using a simplified method.

In engineering practice, in order to significantly improve the bearing capacity of the pile foundation, it is desirable for designers and engineers to embed the pile tip into a harder rock layer if existing, the type of which is commonly referred to as the rock-socketed pile or endbearing pile. Kong et al. [11] presented a mechanical model for the dynamic impedance of a single pile with its pile tip embedded in a bed rock. The dynamic solution for the pile with its pile tip locating on a rock layer was also analyzed by $\mathrm{Wu}$ et al. [12]. However, these research as mentioned above assumed the rock layer to be a rigid solid and has never taken the rock socked depth of the pile into account. In most of geotechnical engineering, the underlying rock layer cannot be completely treated as a rigid material, because the strength of the underlying rock layer maybe lower than the plie material. Therefore, it is of great necessity to study the pile with its pile tip embedded in an elastic rock layer for better understanding the dynamic behaviour of pile foundations.

This paper aims to propose a method to investigate the effects of rock-socketed depth on the vertical vibration characteristics of the pile with its pile tip embedded in an underlying rock layer. The dynamic governing equations of the pile are formulated with aid of FEM, whereas that of the soil are derived from the exact stiffness matrix method (SMM) developed by Ai et 
al. [13]. Based on the compatibility at the pile-soil interface, the dynamic equilibrium equations for the pilesoil interaction problem are established and solved conveniently. Finally, some parametric studies are performed to discuss the influence of rock socketed depth on the dynamic pile impedance.

\section{Pile-soil interaction equations}

The dynamic pile-soil interaction system is described in Fig. 1, where the pile is embedded in a layered medium and subjected to a vertical harmonic dynamic load $p \mathrm{e}^{\mathrm{i} \omega t}$ act its pile head. The pile is commonly considered as one dimensional bar with length $L$, rock-socketed depth $h$, radius $a$, Young's modulus $E_{p}$ and mass density $\rho_{p}$. The material parameters of the soil layer are represented by its Young's modulus $E_{s j}$, Poisson's ratios $\mu_{s j}$, shear modulus $G_{s j}$ and mass density $\rho_{s j}$. Besides, these material parameters with the subscript ' $j$ ' is related to the $j$ th soil layer. Due to the interaction system is under the harmonic vibration condition, all quantities with time factor $\mathrm{e}^{\mathrm{i} \omega t}$ in this paper are suppressed for brevity and $\omega$ denotes the vibration frequency.

In order to establish the pile-soil interaction model, the interaction problem is divided into two parts: (1) the pile is subjected to the contact forces from the foundation at the pile-soil interface and an external axial load at the pile head; (2) the soil is only undergoing the contact forces from the pile at the pile-soil interface. In this paper, FEM is employed to obtain the dynamic governing equation for the pile, which is subdivided into many elements according to the stratification of natural soil and calculation accuracy as shown in Fig. 1. Based on the FEM and 1D bar vibration theory, the dynamic equilibrium matrix equations for a pile can be denoted by:

$$
\mathbf{K}_{p} \mathbf{U}_{p}-\omega^{2} \mathbf{M}_{p} \mathbf{U}_{p}=\mathbf{P}+\mathbf{q}_{p}
$$

where $\mathbf{K}_{p}$ and $\mathbf{M}_{p}$ are the global stiffness matrix and mass matrix of the pile, respectively; $\mathbf{U}_{p}$ and $\mathbf{q}_{p}$ are the global vertical displacement vector and contact force vector of the pile derived from the pile-soil interface, respectively; $\mathbf{P}$ is an external load vector only containing the external load acting at the pile head and other terms are null.

For the soil, the soil flexibility matrices equations can be expressed as:

$$
\mathbf{U}_{s}=\mathbf{D} \mathbf{q}_{s}
$$

where $\mathbf{U}_{s}$ and $\mathbf{q}_{s}$ are the global vertical displacement vector and contact force vector of the foundation derived from the pile-soil interface, respectively; $\mathbf{D}$ is the soil flexibility matrix, the term of which can be obtained through using the exact stiffness matrix method (SMM) developed by Ai et al. [13].
For the sake of completeness, the exact stiffness matrix method for the soil flexibility matrices $\mathbf{D}$ is illustrated as follow in a matrix form:

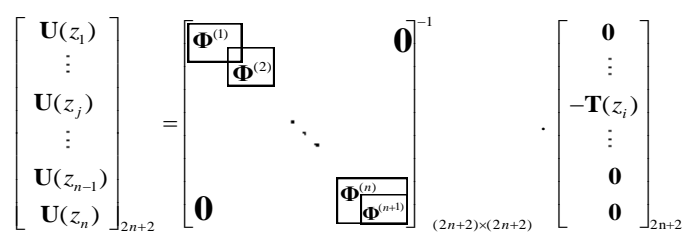

in which $\boldsymbol{\Phi}^{(j)}$ is the stiffness matrix of the soil layer element $j$ deduced from the application of the exact stiffness matrix method to each soil layer element; $\mathbf{U}\left(z_{j}\right)$ represents the displacement vector at the depth of $z_{j}$ in the soil; $\mathbf{T}\left(z_{i}\right)$ is the external load vector acting at the depth of $z_{i}$ in the soil. It should be noted that once the known external load $\mathbf{T}\left(z_{i}\right)$ is determined, the displacement $\mathbf{U}\left(z_{j}\right)$ can be acquired according to equation (3), and $\mathbf{D}$ can be further obtained conveniently. Detail derivation process of Eq. (3) can be referred to Ai et al. [13].

Based on the compatibility and equilibrium at the pile-soil interface, combining Eqs. (1) and (2) yields

$$
\mathbf{U}_{p}=\left(\mathbf{K}_{p}-\omega^{2} \mathbf{M}_{p}+\mathbf{D}^{-1}\right)^{-1} \mathbf{P}
$$

where $\mathbf{P}$ is a known external vector only related to the external dynamic load acting at the pile head, which means the other variables can be achieved conveniently when $p$ is determined.

The vertical displacement of the pile can be achieved by virtue of Eq. (4) and the dynamic response of the soil can be further derived according to Eq. (2). Generally, the dynamic response of the pile is characterized by its dynamic impedance, which is commonly defined as:

$$
K_{v}=p /\left(u_{p 1} G_{s 1} a\right)=\operatorname{Re}\left(K_{v}\right)+\operatorname{Im}\left(K_{v}\right)
$$

where $K_{v}$ is the vertical impedance of the pile representing the true resistance to deformation under dynamic loadings, where the real and imaginary part of $K_{v}$ also stand for stiffness and damping, respectively; besides, $u_{p 1}$ denotes the vertical displacement at the pile head derived from the pile displacement vector $\mathbf{U}_{p}$.

\section{Numerical results and discussion}

\subsection{Comparison with existing solution}

In order to demonstrate the accuracy of the proposed method in this paper, numerical results of the vertical impedance for a pile embedded in a homogeneous elastic half-space are presented in Fig. 2 to compare with the existing results of Zeng and Rajapakse [3], where the following parameters are employed: $E_{p}=1000 E_{s 1}$, $\rho_{p}=1.2 \rho_{s 1}, L=20 a, \mu_{s 1}=0.3$ and $h=0$. According to some published literatures, the normalized vibration 


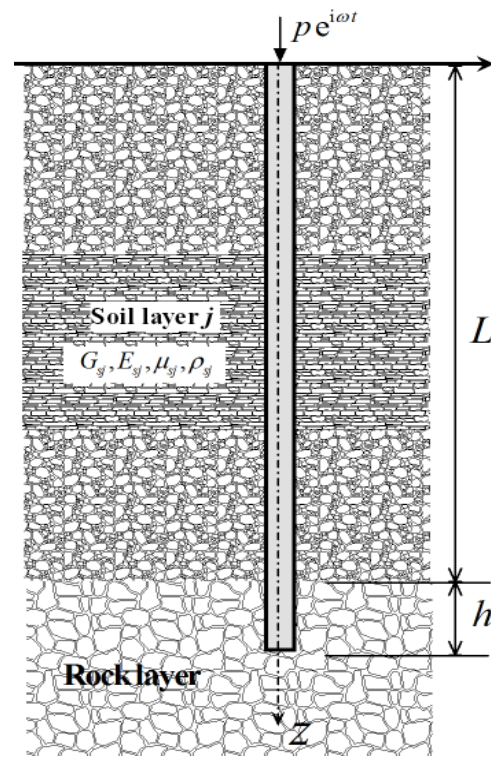

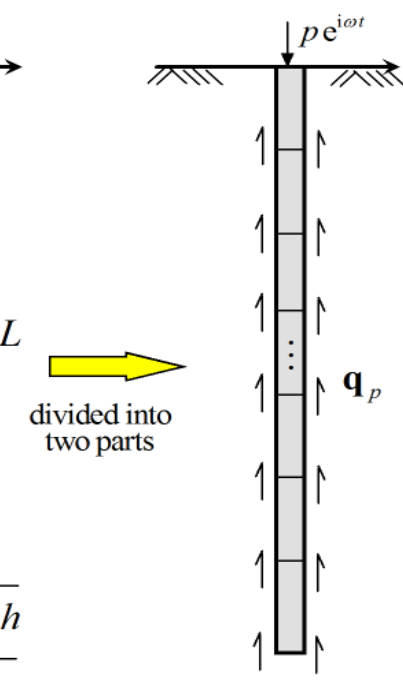

pile model

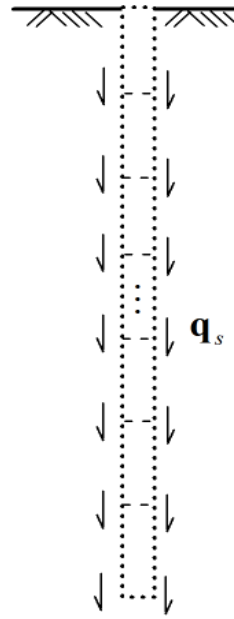

soil model

Fig. 1. Pile-soil interaction system

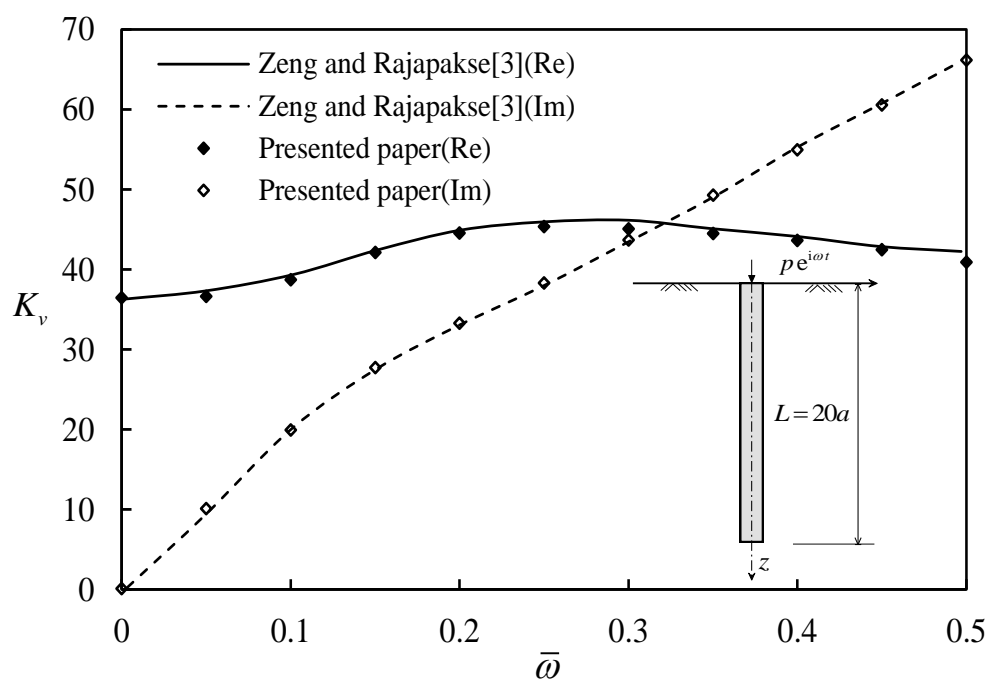

Fig. 2. Comparison of vertical impedance for a pile embedded in a half-space

frequency defined as $\bar{\omega}=a \omega \sqrt{\rho_{s 1} / G_{s 1}}$ are also adopted for brevity. As can be seen from Fig. 2, these results agree well with each other.

\subsection{Effect of rock-socketed depth}

This section aims to investigate the influence of rocksocketed depth on the vertical impedances of a pile with its pile tip embedded in a rock layer. For this purpose, the analysis model with two layer materials are considered: the first one is the conventional soil layer with smaller strength compared with the rock layer; the second one is the rock layer with its strength equal to one hundred times the conventional soil's strength. The relationships among these material parameters are: $E_{p}: E_{\text {rock }}: E_{s 1}=1000: 100: 1, G_{\text {rock }}: G_{s 1}=100: 1, \mu_{\text {rock }}=0.2$, $\rho_{p}: \rho_{\text {rock }}: \rho_{s 1}=1: 1.2: 1.2$ and $\mu_{s 1}=0.3$.In addition, the damping ratio of these two layer is set to be 0.05 for better taking the energy dissipation into account under dynamic conditions. The superscript with "rock" means the material parameters belong to the rock layer.

The vertical impedances in terms of the normalized frequency $\bar{\omega}$ are described in Figs. 3 and 4 with $L=20 a$ and $L=40 a$. Whether for a short pile ( $L=20 a$ ) or for a long pile ( $L=40 a$ ), as illustrated in Figs. 3 and 4, the rock-socketed depth $h$ has marked effect on the real parts (stiffness) of the pile impedance rather than on the imaginary parts (damping). The real parts increase with the increase of the rock-socketed depth $h$ in all frequency ranges, whereas the imaginary parts appear an opposite trend. As $h$ varies from 0 to $4 a$, the variation range of both real and imaginary parts gradually decreases, which reflects that further increase in the rock-socketed depth $(h>4 a)$ may not be effectively improve the pile bearing capacity under dynamic loadings, and $h$ has lees effect on the imaginary parts comparing with the real parts. From an economic point 
of view, adopting the rock-socketed depth $h=2 a$ may be suitable for engineering designer to guarantee the bearing capacity of pile foundations. Besides, a resonance phenomenon is observed: for a short pile ( $L=20 a$ ), the real parts show a sudden decrease but the imaginary parts has a sudden increase when $\bar{\omega}=0.15$; for the long pile ( $L=40 a)$, the same variation also occurs when $\bar{\omega}=0.05$, which is due to the vibration frequency approaching the natural frequency of the pilesoil interaction system .

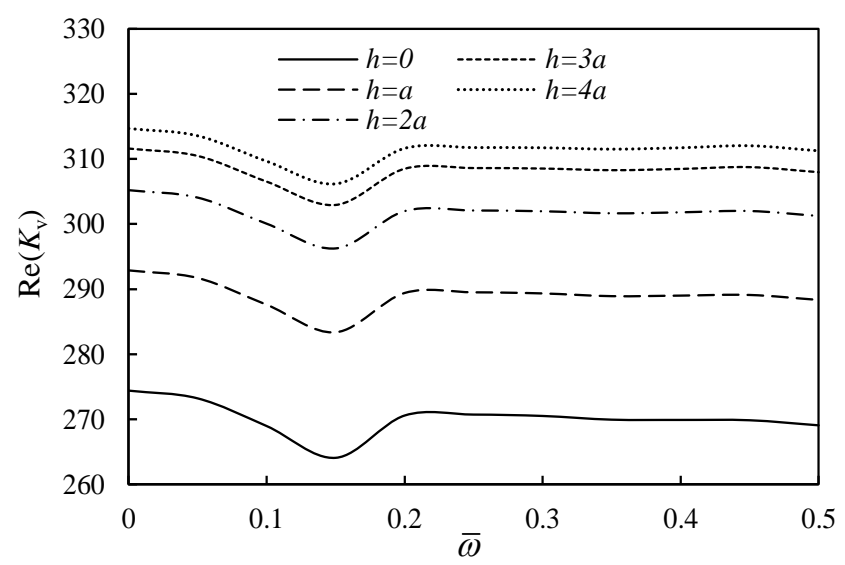

(a) Real parts of vertical impedances

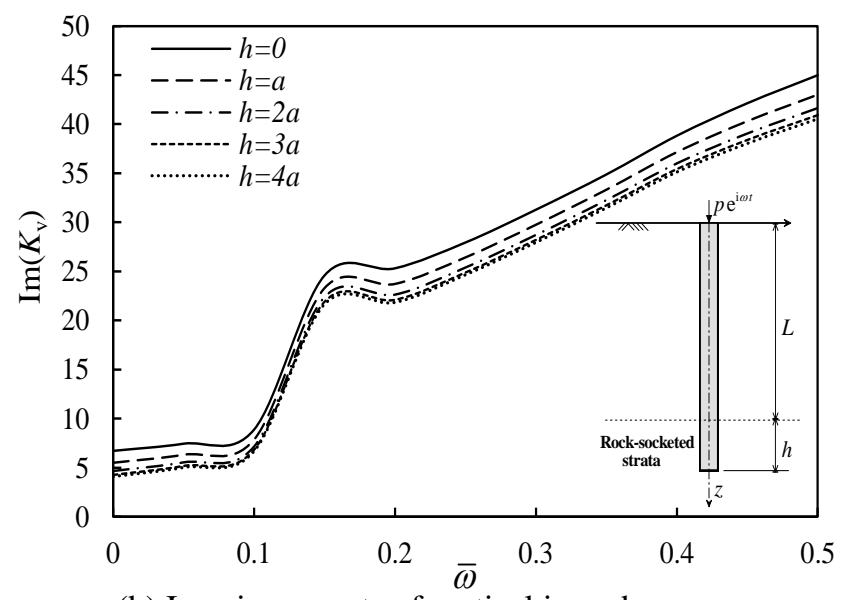

(b) Imaginary parts of vertical impedances

Fig. 3. Vertical impedances of a single pile with different rock-socketed depth $(L=20 a)$

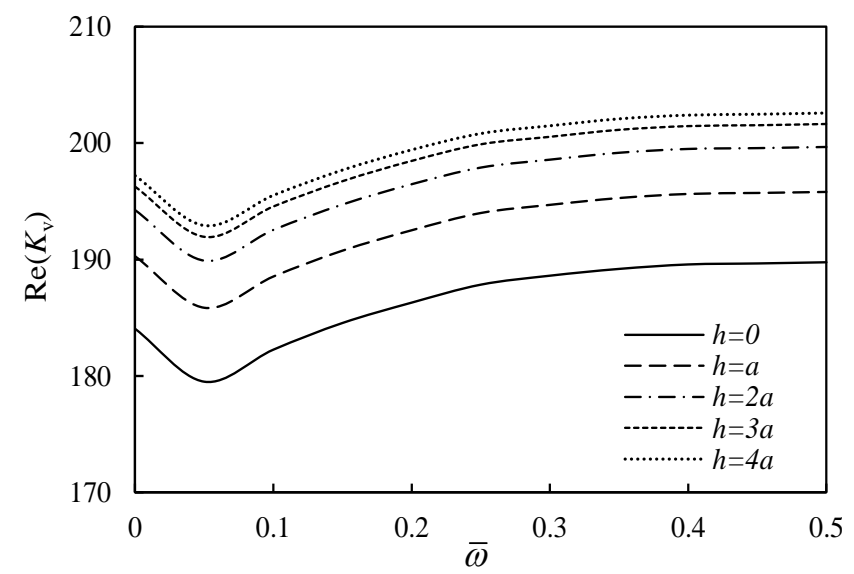

(a) Real parts of vertical impedances

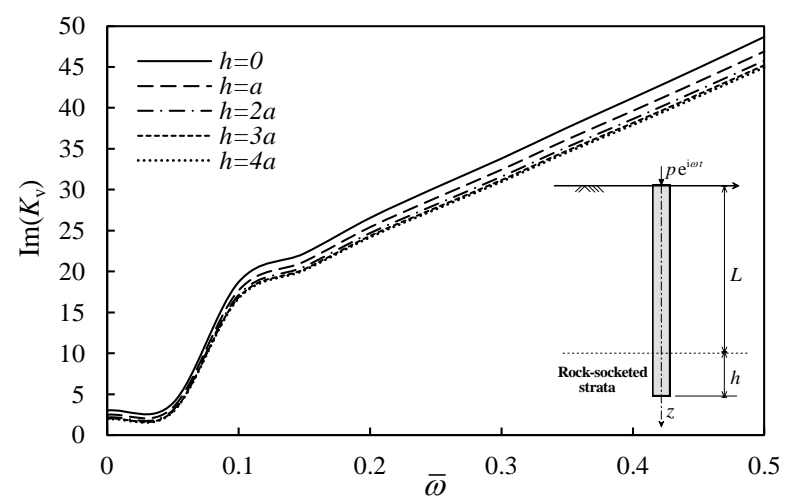

(b) Imaginary parts of vertical impedances

Fig. 4. Vertical impedances of a single pile with different rock-socketed depth $(L=40 a)$

\section{Conclusion}

A simplified method has been proposed in this paper to investigate the influence of rock-socketed depth on the vertical impedance of an embedded pile. The pile is modelled as a one-dimension bar and the surrounding medium as three-dimension isotropic body. Based on the FEM and SMM, the pile-soil interaction problem is solved and then a parameter study corresponding to the rock-socketed depth is carried out. Numerical results indicate that the vertical impedance of pile shows an obvious dependence on the rock-socketed depth when $h \leq 2 a$, especially for the real parts (dynamic stiffness). A resonance phenomenon occurs and the amplitude of the vertical impedance decreases when the vibration frequency is close to natural frequency of the interaction system.

\section{Acknowledgments}

The authors acknowledge the financial support from National Natural Science Foundation of China (Nos.51908225, 51678171), China Postdoctoral Science Foundation (2019M652899, 2019M662917, 2019M662918), Postdoctoral International Training Program of Guangzhou and the Peal River S \& T Nova Program of Guangzhou (201806010095).

\section{References}

1. R.K.N.D. Rajapakse, A.H. Shah, International Journal of Solids and Structures Vol. 23, p. 267 (1987)

2. R.K.N.D. Rajapakse, A.H. Shah, Soil Dynamic and Earthquake Engineering Vol. 8, p. 145 (1989)

3. X. Zeng, R.K.N.D. Rajapakse, Journal of Engineering Mechanics Vol. 125, p. 1048(1999)

4. J.F. Lu, B. Xu, J.H. Wang, D.S. Jeng, Acta Mechanica Vol. 207, p. 29(2009)

5. W. Liu, M. Novak, Earthquake Engineering and Structural Dynamics Vol. 23, p. 1239 (1994) 
6. T. Zheng: Acta Mechanica Sinica Vol. 13, p. 241(1997)

7. P.L.A. Barros: 16th ASCE Engineering Mechanics Conference, University of Washington, Seattle, July 16-18 (2003)

8. M.K. Kim, J.S. Lee, M.K. Kim: KSCE Journal of Civil Engineering Vol. 8, p. 221 (2004)

9. K.H. Wang, W.B. Wu, Z.Q. Zhang, C.J. Leo: Computers and Geotechnics Vol. 37, p. 536 (2010)
10. Z.Y. Ai, Z.X. Li, N.R. Cang: Soil Dynamics and Earthquake Engineering Vol. 60, p. 22(2014)

11. D. Kong, M. Luan, X. Ling: Journal of Harbin Institute of Technology(New Series) Vol. 15, p. 393(2008)

12. W.B. Wu, K.H Wang, Z.Q Zhang, et al.: International Journal for Numerical and Analytical Methods in Geomechanics Vol. 37, p. 2860 ( 2013)

13. Z.Y. Ai, Z.X. Li, N.R. Cang: Soil Dynamics and Earthquake Engineering Vol. 60, p. 22 (2014 